\title{
Éléments pour un examen critique des études neurodidactiques : point de vue de didacticiens des sciences et ouverture vers un dialogue
}

Evaluation of neurodidactic studies by didacticians of science: openings for an interdisciplinary dialogue

\section{Claude Caussidier et Grégoire Molinatti}

\section{OpenEdition Journals}

Édition électronique

URL : https://journals.openedition.org/educationdidactique/2998

DOI : 10.4000/educationdidactique.2998

ISSN : 2111-4838

\section{Éditeur}

Presses universitaires de Rennes

\section{Édition imprimée}

Date de publication : 28 août 2018

Pagination : $59-78$

ISBN : 978-2-7535-7554-7

ISSN : 1956-3485

\section{Référence électronique}

Claude Caussidier et Grégoire Molinatti, «Éléments pour un examen critique des études neurodidactiques : point de vue de didacticiens des sciences et ouverture vers un dialogue », Éducation et didactique [En ligne], 12-1 | 2018, mis en ligne le 28 août 2020, consulté le 23 août 2022. URL : http:// journals.openedition.org/educationdidactique/2998; DOI : https://doi.org/10.4000/ educationdidactique.2998 


\title{
ÉLÉMENTS POUR UN EXAMEN CRITIQUE DES ÉTUDES NEURODIDACTIQUES : POINT DE VUE DE DIDACTICIENS DES SCIENCES ET OUVERTURE VERS UN DIALOGUE
}

\author{
Claude Caussidier \\ Composante Didactique et Socialisation du LIRDEF (EA 3749), \\ université de Montpellier, DR2 CNRS \\ Grégoire Molinatti \\ Composante Didactique et Socialisation du LIRDEF (EA 3749), \\ université de Montpellier, MCF en sciences de l'éducation et de la communication, \\ Chercheur associé au Centre Norbert Elias (UMR 8562), Marseille
}

\begin{abstract}
Dans cet article, nous discutons des possibilités d'un dialogue interdisciplinaire entre didactique et neurodidactique des sciences, au regard des partis pris épistémologiques propres à chacun de ces champs d'investigation de l'apprentissage des sciences. Pour ce faire, nous analysons un corpus constitué de publications et de communications récentes en neuroéducation et en neurodidactique des sciences en explicitant les cadres théoriques mobilisés, les questions de recherche et les méthodes d'étude mises en œuvre. Nous montrons comment les neurosciences de l'éducation peuvent concevoir les questions d'apprentissage sous l'angle de comparaisons normal / pathologique et discutons des conceptions du sujet que ces comparaisons sous-tendent. Nous distinguons ensuite trois catégories de travaux en neurodidactique des sciences et discutons des réductionnismes opérés et des dérives normatives possibles, en interrogeant notamment la prise en compte des enjeux et des contextes d'éducation scientifique, ainsi que la place dévolue aux interactions langagières dans les apprentissages.
\end{abstract}

Mots-clés : neuroéducation, didactique des sciences, théorie du sujet, normes.

Evaluation of neurodidactic studies by didacticians of science: openings for an interdisciplinary dialogue

In this paper, we discuss the possibilities of an interdisciplinary dialogue between the didacticians of science and the neurodidacticians, with regard to the epistemological biases relevant to each of these fields of investigation of science learning. With this aim, we analyzed a corpus set up from recent publications and communications in neuroeducation and science neurodidactics (brain-based learning) by exposing the mobilized theoretical frames, the research questions, and the implemented methods of study. We show how the brain-based learning research can conceive the questions of learning with a normal versus pathological perspective, and we discuss the underlying concepts of the subject implied by this simplifying dichotomy. Then, we characterize three categories of studies in science neurodidactics and discuss their respective reductionisms and possible normative drifts, taking into account their specific science education contexts and stakes, as well as the place attributed to the linguistic interactions in the science learning processes.

Keywords: neuroeducation, didactics of science, theory of the subject, norms. 


\section{INTRODUCTION}

Les sciences de l'éducation et la didactique des sciences ne sont pas les seules disciplines dont les questions de recherche sont saisies par les neurosciences. On peut considérer que ce phénomène touche une bonne partie des sciences humaines et sociales, domaines juridiques, domaines de la psychologie sociale, du marketing, des rapports sociaux de genre ou de l'éthique ${ }^{1}$. Dès leur émergence, les neurosciences se sont développées en mettant en avant une promesse, ou plutôt une double promesse, à la fois de compréhension et de thérapeutique ou d'application. Il est donc compréhensible que des études récentes qui questionnent l'émergence des neurosciences sociales puissent se donner un horizon de démystification au regard des mouvements de fascination, ou au contraire de rejet inconditionnel, qu'elles peuvent provoquer au sein des sciences humaines, par leur capacité potentielle à féconder, à révolutionner, ou au contraire à coloniser voire à dissoudre les champs de recherche concernés. Loin de rester confiné au monde académique, le biologisme (discours reposant sur une lecture biologique du monde social) jouit d'un regain de popularité, notamment à travers la médiation des neurosciences (presse, édition d'auteurs scientifiques) et leurs apports à la compréhension du développement personnel (Lemerle, 2011).

Depuis leur constitution en champ de recherche pluridisciplinaire et autonome dans les années 1960 aux États-Unis, et un peu plus tard en France, les sciences prenant le cerveau, son développement et son fonctionnement, pour objet ont connu un essor considérable. Soutenues au cours des dernières décennies par des grands projets nationaux et internationaux, les neurosciences ont bénéficié du développement de nouvelles techniques d'exploration au premier rang desquelles figurent les techniques dites non invasives d'imagerie cérébrale. Elles ont dès lors proposé de ré-explorer les fondements neuronaux des grands processus cognitifs (mémoire, apprentissage, langage, raisonnement, empathie, sociabilité, conscience...) essayant de relier les phénomènes neuronaux et les entités cognitives décrites par la psychologie cognitive (Tiberghien, 2007). Un nouveau domaine de recherche, celui des sciences cognitives, a ainsi émergé. Ces dernières rassemblent les contributions de plusieurs disciplines, telles que la psychologie cognitive, la psychologie du compor- tement, la linguistique, les neurosciences, la philosophie, l'ethnologie, l'anthropologie, la sociologie, l'intelligence artificielle, les mathématiques, l'informatique. À l'intérieur des sciences cognitives, les neurosciences cognitives forment une discipline ayant pour objectif de comprendre les mécanismes neurobiologiques qui sous-tendent la cognition (perception, motricité, langage, mémoire, raisonnement, émotions...). Elles font appel pour une large part à l'imagerie cérébrale, à la stimulation de zones corticales et à la modélisation.

Les neurosciences cognitives proposent aujourd'hui des éclairages, et des possibilités de régulations sur des questions sociales (économie, justice), dont la question éducative. Les chercheurs en neurosciences cognitives pensent ainsi parvenir à une compréhension des processus cognitifs qui déboucherait sur des possibilités d'applications en éducation. Le champ des neurosciences de l'éducation (educational neuroscience) s'est alors constitué, visant d'emblée des débouchés pratiques qui forment le champ de la neuroéducation.

La neuroéducation, aussi appelée brain-based learning (Hardiman et al., 2011) dans les pays anglophones, s'intéresse plus particulièrement à l'amélioration de l'apprentissage et, en présence de troubles de celui-ci, à leur remédiation en considérant les fonctions mentales en tant que processus de traitement et de production d'informations (attention, mémoire, langage, etc.). Ces dernières années les travaux de neurocognition rendent aussi compte de processus cérébraux impliqués dans des fonctions plus complexes (affectivité, pensée mystique, manipulation de symboles et de concepts, apprentissage de connaissances...). Par exemple, la neurodidactique se donne comme ambition non seulement de comprendre les bases biologiques de processus didactiques, tels que le changement conceptuel mais aussi à mettre en œuvre des techniques d'application chez les élèves (Dunbar et al., 2007).

Ainsi la neurodidactique, comme la neuroéducation, prescrit des méthodes d'enseignement, d'organisation et de planification des leçons et programmes scolaires basés sur les travaux des neurosciences cognitives, en prenant en compte des facteurs tels que le développement cognitif, et la maturation sociale et émotionnelle. Ces deux disciplines sont basées sur la croyance générale que l'apprentissage peut être accéléré et amélioré si les éducateurs construisent leurs enseignements à la lumière des découvertes des 
neurosciences cognitives plus que sur des pratiques éducatives antérieures ${ }^{2}$.

Prenant acte de l'émergence de ce champ de recherche, nous nous sommes posé, en didacticiens des sciences ${ }^{3}$, la question des possibilités d'un dialogue avec des chercheurs en neuroéducation, sans présumer a priori de l'impossibilité de ce dialogue. Si les possibilités d'un tel dialogue nous semblent, à l'heure où nous écrivons ce texte, bien limitées, nous ambitionnons d'expliciter en quoi. Car force est de constater que, à notre connaissance, les principales revues de didactique des sciences, $\mathrm{du}$ moins francophones, font pour ainsi dire l'impasse du sujet neurobiologique, sans pour autant en ré-expliciter les raisons. Il nous semble nécessaire, à l'aune du développement des neurosciences, de renouveler ce débat. Nous souhaitons modestement $\mathrm{y}$ apporter notre contribution.

Il s'agit donc d'exprimer notre point de vue sur les présupposés épistémologiques qui structurent les recherches en neuroéducation et en neurodidactique des sciences. Cet objectif passe par la définition que ces recherches donnent (de manière implicite ou explicite) du sujet en situation d'apprentissage, au regard de quels enjeux éducatifs.

Dans cette perspective, nous revenons tout d'abord sur les principales théories qui, au cours de l'histoire, ont proposé de relier cerveau et apprentissage. Il est question de préciser dans quelle mesure les théories éducatives ont pu s'articuler ou dialoguer avec des approches matérialistes du fonctionnement du système nerveux, pour essayer de saisir les raisons de la rupture d'un tel dialogue. Dans un second temps, nous proposons une lecture des principaux travaux dans le champ de la neuroéducation en nous focalisant sur leurs contributions à la compréhension des phénomènes d'apprentissage. Enfin, nous analysons plus spécifiquement les travaux de neurodidactique des sciences pour tenter de dégager les présupposés épistémologiques qui les sous-tendent. Ce travail nous permet de discuter des fondements de la normativité de la neuroéducation et de la neurodidactique des sciences ainsi que des réductionnismes opérés par ces disciplines émergentes, notamment sur le processus d'apprentissage conceptuel. Il invite les didacticiens à clarifier leurs propres positionnements épistémologiques, notamment la manière dont ils prennent en compte la dimension neurobiologique du sujet engagé dans des apprentissages en sciences.

\section{CERVEAU ET APPRENTISSAGE : PERSPECTIVES HISTORIQUES}

La question de la localisation organique de fonctions telles que la perception, la commande du mouvement ou la mémoire est ancienne. Les thèses céphalocentriques ou crâniocentristes ont été proposées dès l'Antiquité, et celles des localisations cérébrales ventriculaires ont émergé dans le monde Arabe, puis en Occident, au Moyen-Âge. À la Renaissance, le renouveau de l'anatomie, illustré par la Fabrica de Vésale (1543), puis les travaux de Descartes (De Homine, 1662) rendent compte d'une tendance à localiser, les fonctions sensorielles et motrices, en cohérence avec une conception mécaniste et dualiste.

Pour autant l'inscription cérébrale des fonctions mentales, à comprendre comme une rupture épistémologique vis-à-vis du dualisme cartésien, ne commence à être construite dans des termes proches de ceux qui vont être repris au sein des neurosciences modernes, qu'avec les travaux du groupe londonien de la Royal Society et notamment de Willis. Willis développe, dans son Cerebri anatome (1664), une conception «solidiste », moniste, où les fonctions mentales sont assurées par des structures cérébrales: le cervelet est le siège principal de la pensée rationnelle chez l'homme et de la pensée sensible chez les animaux ${ }^{4}$. Mobilisant des arguments cliniques et d'anatomie comparée, il propose également des spécialisations régionales fonctionnelles, notamment pour ce qui concerne la mémoire et l'apprentissage qu'il situe au niveau du cortex cérébral. Cette pensée localisationniste a été largement popularisée, et de manière radicale souvent, par les théories phrénologiques introduites par Gall avec la publication en 1810 de son Anatomie et physiologie du système nerveux en général et du cerveau en particulier, "Sur les fonctions du cerveau et sur celles de chacune de ses parties, avec des observations sur la possibilité de reconnaître les instincts, les penchants, les talents, ou les dispositions normales et intellectuelles des hommes [...] par la configuration de leur cerveau et de leur tête ». Parmi ces fonctions, considérées comme innées, certaines concernent l'apprentissage et l'éducation. C'est le cas de la « capacité à recevoir une éducation » (Wyhe, 2002) qui est localisée dans une région frontale tout comme l'aptitude à relier les nombres entre eux, ancêtre de la « bosse des maths ». 
C'est donc au tournant du XVIII ${ }^{\mathrm{e}}$ siècle que se dessine réellement le matérialisme consistant à attribuer au système nerveux en général, et au cerveau en particulier, un rôle déterminant comme support organique des fonctions de l'âme et des comportements, dont l'apprentissage. Dès cette période, le courant matérialiste se polarise sur la recherche des déterminismes des fonctions cérébrales et entre en résonance avec un débat philosophique et social relatif à la liberté humaine et au déterminisme. Ainsi pour Fisher (2001), c'est au XIX ${ }^{\mathrm{e}}$ siècle qu'émerge « cette façon de penser le vivant à partir de cette alternative entre déterminisme génétique et autonomie du développement $»$. Trois grands courants relativement autonomisés, qui s'inscrivent alors au sein du paradigme moniste matérialiste, vont participer à ce débat (voir Molinatti, 2007 pour une présentation détaillée de ces courants de pensées et des auteurs et ouvrages auxquels il est fait référence) :

- les approches anatomo-physiologiques (physiologie réflexologique de Descartes, de La Mettrie, Hartley \& Whytt, puis Bell \& Magendie) ou de psychologie (électro)physiologiques (Setchenov, Thorndike \& Pavlov) considèrent l'organisme selon des réponses stéréotypées aux variations du milieu, tout en comprenant l'épigenèse comme une liberté du vivant à répondre, par la régulation, aux facteurs du milieu ;

- les approches génétiques (thèses préformistes de Weissman puis Bateson, Sutton, Johansen) qui tendent à faire admettre l'autonomie du vivant par rapport au milieu, et de la biologie du développement (école d'embryologie expérimentale de Driesh, Spemann \& Mangold) ;

- les approches éthologiques et psychologiques des déterminismes du fonctionnement cérébral.

Au sein de ces trois approches on peut distinguer les conceptions phylogénétiques des tenants de la psychologie comparée (Spencer, Romanes puis Morgan) et les conceptions défendant un déterminisme génétique strict (de l'hérédité des facultés mentales de Galton au darwinisme psychologique d'Haeckel, fondations idéologiques de l'eugénisme et du racisme scientifique). On peut également distinguer les conceptions behavioristes (dont le manifeste est formulé en 1913 par Watson) pour lesquels « le milieu se trouve investi de tous pouvoirs vis-à-vis des individus » (Canguilhem, 1989), qui seront radica- lement remises en cause par les concepts de représentation ou d'image mentale introduits par Lashley et Tolman avec leurs « cartes cognitives » proposant ainsi un "retournement du rapport organisme / milieu » selon lequel l'organisme se «propose au monde " (Canguilhem, 1989). Enfin, à partir des années 1930, et en partie en opposition au modèle objectiviste développé par Lorenz (l'instinct comme prédisposition innée à apprendre), s'affirme la théorie constructiviste introduite par Maier et Schneirla, qui met en avant l'indissociabilité des déterminismes génétiques et épigénétiques dans l'explication des comportements. C'est dans la continuité de ce dernier courant constructiviste que l'on peut situer les fondateurs de la psychologie génétique, en particulier Vygotski et Piaget. Il est à noter que Piaget (1967) a la volonté de tracer une continuité entre réalité biologique et réalité cognitive. Pour Piaget, la connaissance est ainsi en partie conçue comme un produit de l'adaptation de la pensée au réel, donc selon une conception empruntant aux approches biologique et évolutionniste.

Ces courants reliant de façon plus ou moins stricte biologie et pensée font apparaître trois caractéristiques relatives au statut du Sujet dans les approches scientifiques des apprentissages: premièrement cette question a d'abord été prise en charge par les sciences de la nature matérialistes; deuxièmement, elle a été aux prises avec des débats idéologiques (inné / acquis, préformiste / environnementaliste, génétique / épigénétique) comme l'a souligné Jacob (1981) et, enfin, certains des fondateurs de la psychologie génétique dont se réclament les sciences de l'éducation et la neurodidactique des sciences maintiennent le lien entre sujet biologique et sujet cognitif.

Force est de constater que les grands courants de pensée qui structurent aujourd'hui le champ de la didactique des sciences, définie comme "science qui étudie, pour un domaine particulier les phénomènes d'enseignement, les conditions de transmission de la "culture" propre à une institution scientifique et les conditions de l'acquisition des connaissances » (Joshua \& Dupin, 1999) n'ont pas maintenu la continuité entre sujet biologique et sujet cognitif. En effet, les principaux cadres de pensée (Simonneaux, 2011) mobilisés en didactique des sciences, du moins en France, envisagent le sujet cognitif exclusivement sous l'angle épistémologique, anthropologique ou psychosocial. Ainsi les théories anthropologiques du 
didactique (la transposition didactique proposée par Chevallard en 1985, la théorie des situations proposée par Brousseau en 1998, la théorie de l'action conjointe proposée par Sensevy \& Mercier en 2007, le concept de schème développé par Vergnaud en 1994, les travaux inspirés de la psychologie sociale sur les conceptions [système « Knowledge, Values, Practices » proposé par Clément en 2004], et la théorie des changements conceptuels inspirés de l'épistémologie des sciences par Posner, Strike, Hewson, et Gertzog en 1982) ne mobilisent pas de dimension biologique dans leurs théories du Sujet en situation d'apprentissage. Ces courants de pensée partagent une vision de l'apprentissage inscrite dans un paradigme constructiviste, dans son acception personnelle ou sociale, c'est-à-dire comme une construction cognitive contextualisée, le plus souvent volontaire, consciente et explicite.

On peut avancer deux hypothèses non exclusives pour tenter de comprendre pourquoi les courants de pensée en didactique ont fait l'économie du sujet biologique. D'une part, comme cela a été le cas pour une grande partie des sciences humaines, la didactique des sciences, de constitution récente, s'est tenue à distance des dérives idéologiques associées aux tentatives de naturalisation des questions psychologiques et sociales. Il s'est agi de décontaminer le sujet cognitif de tous les déterminismes biologiques qui avaient pu s'exprimer de manière radicale dans les idéologies racialistes, eugénistes ou sexistes. D'autre part, une partie au moins des spécialistes de l'apprentissage humain semble s'être accommodée d'un sujet biologique " minimal » caractérisé par ses propriétés de plasticité cérébrale, support biologique de l'apprentissage. Ainsi, le modèle neurobiologique dit du calendrier cérébral (Edelman, 1987 ; Changeux, 1983) qui tente une synthèse reposant sur l'interaction entre facteurs environnementaux, grâce aux propriétés de neuroplasticité, et facteurs génétiques dans la réalisation du phénotype cérébral apparaît compatible avec tout à la fois le paradigme constructiviste et une pensée humaniste.

C'est en prenant en considération ces filiations et ces ruptures épistémologiques que nous nous proposons d'analyser des travaux dans les champs émergents de la neuroéducation et de la neurodidactique des sciences.

\section{« COMPRENDRE LE CERVEAU : NAISSANCE D'UNE SCIENCE DE L'APPRENTISSAGE' " OU LES FONDEMENTS THÉORIQUES DE LA NEUROÉDUCATION}

Comme nous l'avons vu en introduction, la neuroéducation est basée sur les neurosciences de l'éducation, se revendiquant elles-mêmes des neurosciences cognitives, qui explorent au niveau biologique les différences, et surtout les ressemblances interindividuelles dans l'anatomie du cerveau et dans ses modalités de fonctionnement. Sur cette base, des applications visent à la mise en œuvre de programmes et de technologies éducatives pour aider à l'apprentissage. Dans ce paragraphe nous proposons de faire le point sur les données des neurosciences cognitives qui trouvent une application dans le domaine de l'apprentissage ainsi que leurs possibles utilisations à partir des travaux les plus significatifs. Nous dégagerons les traits principaux du sujet apprenant auquel elles s'adressent, voire qu'elles participent à construire.

\section{Les neurosciences cognitives et l'apprentissage}

Les neurosciences cognitives ont fourni de très nombreuses données concernant les bases physiologiques de l'apprentissage, notamment depuis le développement des techniques de neuroimagerie fonctionnelle. Par exemple, Damasio (2001) avance que les émotions jouent un rôle important dans l'ensemble des processus de traitement des données par le cerveau. Ainsi, le recours d'un individu à une logique déductive (rationnelle, sans implication personnelle ou sociale) fait appel à une zone du cerveau impliquée dans les émotions et les sentiments (Houdé et al., 2001).

En 2005, Atherton posait les questions suivantes concernant les éclairages que l'on pouvait demander aux neurosciences cognitives : comment la mémoire, la perception, le raisonnement et les émotions sontils représentés dans le cerveau ? Quelles sont les relations entre cognition et émotion? Nous allons voir ci-dessous les réponses apportées par les neurosciences cognitives dans des domaines qui touchent aux apprentissages scolaires (mémoire, attention sélective, intelligence, apprentissages de la lecture et des mathématiques). 


\section{La mémoire}

La mémoire peut être favorisée par la mise en jeu de diverses fonctions (sommeil, vision, audition) et c'est un phénomène dynamique (Eustache $\&$ Guillery-Girard, 2016). Les expérimentations montrent que la stimulation des zones cérébrales spécifiques intervenant dans la mémoire conduisent à une consolidation de celle-ci. Ainsi, des stimulations magnétiques transcrâniennes du cortex cérébral, préalablement à une tâche d'apprentissage moteur (poursuivre une cible visuelle mouvante sur un écran avec une manette de jeu), consolident un comportement moteur simple, appris (Boyd $\&$ Linsdell, 2009). Les sujets soumis aux stimulations font ensuite moins d'erreurs de poursuite que les sujets contrôles. Ces résultats indiquent qu'un phénomène de consolidation de la mémoire motrice a eu lieu, un processus au cours duquel l'information d'abord stockée momentanément dans la mémoire à court terme passe dans la mémoire à long terme, plus stable et robuste.

Wang et al. (2014) ont utilisé des stimulations magnétiques transcrâniennes non invasives pour stimuler les réseaux cortico (cortex pariétal latéral)-hippocampiques, supposés intervenir dans la mémoire associative, chez l'homme. Ils montrent que plusieurs sessions de stimulation augmentent la connectivité dans ces réseaux mesurée par IRMf (imagerie par résonance magnétique fonctionnelle) ainsi que les performances de la mémoire associative (mesurée via des tâches de rappel d'associations arbitraires entre visages et mots). Ces changements persistent environ 24 heures après la stimulation. Toutefois, bien que les auteurs soulignent que cette technique puisse être considérée dans le domaine de la cognition, ils ne l'envisagent que potentiellement utile dans les cas de lésions conduisant à des troubles de la mémoire.

Des tâches cognitives plus complexes peuvent aussi être améliorées par stimulation. Ainsi, Flöel et al. (2008) ont réalisé une stimulation électrique directe de zones spécifiques du cerveau sur des sujets au cours de leur acquisition d'un lexique de 30 mots en langue étrangère. L'apprentissage est de type associatif : le sujet doit réaliser l'appariement correct entre un objet usuel présenté à la vue et un nouveau mot en langue étrangère. La rapidité et l'efficacité de l'apprentissage ainsi que le transfert du mot étranger dans la langue native sont mesurés à la fin de la session d'apprentissage. Les sujets soumis à cette stimulation montrent un apprentissage plus rapide et meilleur que les sujets témoins, cet effet n'étant plus significatif après une semaine. Les auteurs en déduisent cependant une efficacité de cette technique de stimulation pour améliorer l'apprentissage du langage. La technique peut en effet être améliorée non seulement en optimisant les paramètres de stimulation mais aussi en l'associant à des tâches cognitives ad-hoc.

Snowball et al. (2013) ont montré l'efficacité d'une stimulation non-invasive du cerveau sur l'apprentissage des mathématiques. Les auteurs utilisent une stimulation transcrânienne par bruit aléatoire pour activer le cortex préfrontal dorsolatéral bilatéral (DLPFC, zone clef dans le traitement des mathématiques) dont ils enregistrent simultanément l'activité hémodynamique. Cinq jours de stimulations couplées avec un apprentissage arithmétique induisent une augmentation de la rapidité de calcul et de l'apprentissage arithmétique basé sur la mémoire, chez les sujets traités. Ces améliorations comportementales sont associées à une activité vasculaire spécifique du DLPFC gauche. Ces changements de comportement persistent six mois après la stimulation transcrânienne. Ces résultats indiquent donc qu'une stimulation transcrânienne adaptée peut induire des améliorations à long terme des capacités cognitives.

Cependant des travaux du même groupe (Iuculano et al., 2013) indiquent que cette amélioration de l'apprentissage pourrait avoir des conséquences non désirées. Ainsi les expériences de Iuculano montrent que la stimulation transcrânienne du cortex pariétal postérieur facilite l'apprentissage numérique tandis qu'elle perturbe l'automaticité quant à l'utilisation de ce même matériel numérique. La stimulation du cortex préfrontal dorsolatéral produit l'effet inverse. Les auteurs soulignent donc que l'amélioration de l'apprentissage par ce type de technique peut avoir lieu aux dépends d'autres fonctions cognitives, ce qui devrait réduire leur utilisation chez des sujets sains.

\section{L'attention sélective}

En ce qui concerne l'attention sélective, trois réseaux fonctionnels traitant ses trois sous-fonctions principales ont été identifiés. Un réseau traite 
principalement les fonctions d'alerte (maintien d'un état de grande sensibilité aux stimuli entrants), un autre celles d'orientation (sélection des informations venant des systèmes sensoriels), et un troisième les fonctions de contrôle de l'exécution (mécanismes de suivi et de résolution des conflits entre les pensées, les émotions et les réponses) (Posner, Rothbart, \& Rueda, 2011). L'attention sélective peut ainsi être considérée comme un système avec sa propre organisation fonctionnelle.

Jacobson et al. (2012) ont tenté de moduler, par stimulation transcrânienne, l'activation des substrats corticaux de l'attention pendant un apprentissage. Dans leur protocole expérimental, cette modulation est basée sur la mise en activité des substrats cérébraux qui seraient responsables de l'attention sélective tandis que l'on réduit l'activité des substrats cérébraux possiblement impliqués dans la détection des changements d'orientation dans l'environnement, la mise en activité de cette dernière zone étant susceptible de diminuer l'efficacité de l'attention sélective. Pour cela, les auteurs appliquent simultanément une stimulation facilitatrice, qui va donc activer les circuits, sur l'ensemble des zones responsables de l'attention sélective (sulcus intrapariétal gauche / cortex pariétal supérieur) et une stimulation inhibitrice, qui va donc réduire l'activité des circuits, sur la zone responsable de la fonction de détection des changements d'orientation (cortex pariétal inférieur droit), pendant une tâche de reconnaissance verbale de mots. Ce protocole de stimulation entraîne une amélioration de la mémorisation du matériel verbal et a donc un effet facilitateur sur la mémoire épisodique. Les auteurs réalisent donc une mise en jeu différentielle des trois réseaux de l'attention au profit $\mathrm{du}$ renforcement de l'attention sélective, manifestée par une amélioration de la mémoire épisodique. Selon les auteurs, ce type de protocole pourrait avoir des utilisations pratiques pour développer des interventions en vue d'améliorer les aptitudes de personnes souffrant de déficits attentionnels.

\section{L'intelligence}

Les travaux réalisés par Houdé ${ }^{6}$ (2005) sur la construction de l'intelligence le conduisent à définir différemment que ne l'ont fait Piaget et les néopiagéciens, les stades du développement de l'intelligence chez l'homme ainsi que les mécanismes de tran- sition d'un stade à l'autre. Houdé est en désaccord avec le modèle par paliers de Piaget décrivant que le développement cognitif de l'enfant s'exécute selon un processus linéaire et cumulatif, et pense que ce modèle doit être réexaminé pour deux raisons. D’une part, il existe déjà chez le bébé des capacités cognitives assez complexes, des connaissances physiques, logiques et mathématiques, ainsi que psychologiques non réductibles à un fonctionnement strictement sensori-moteur (Girotto \& Gonzalez, 2008). Ainsi d'après ces auteurs, dès l'âge de 6 mois, un bébé pourrait saisir le sens des mots les plus courants même s'il ne sait pas encore les exprimer et il pourrait donc comprendre le langage. D'autre part, toujours d'après eux, la suite du développement de l'intelligence jusqu'à l'âge adulte est jalonnée d'erreurs de logique, de biais perceptifs non prédits par la théorie piagétienne. Plutôt que de suivre un programme continu qui mènerait, sans retour en arrière, du stade sensorimoteur à l'abstraction, l'intelligence de l'enfant avancerait de façon discontinue avec remaniements et transformations d'un type d'activité initialement prépondérant, et subissant alors une diminution, voire même une disparition, au profit d'un autre type d'activité.

Cependant, dans une étude réalisée en 2011, Houdé et al. montrent aussi que les stades cognitifs et comportementaux décrits par Piaget ont un substrat cérébral. Ces auteurs réalisent une expérimentation au cours de laquelle ils enregistrent, en imagerie par résonance magnétique fonctionnelle (IRMf $\left.{ }^{7}\right)$, l'activité cérébrale de deux groupes d'enfants auxquels on soumet la tache de conservation des nombres de Piaget. Un groupe est constitué d'enfants ne conservant pas les nombres d'après la théorie de Piaget (sauf par intuition visiospatiale, 5-6 ans), l'autre groupe conservant les nombres (9-10 ans). Les changements cognitifs permettant aux enfants d'acquérir la conservation des nombres apparaissent être reliés à la contribution d'un réseau neuronal pariétofrontal bilatéral impliqué dans les fonctions numériques et exécutives.

Par ailleurs, Houdé $(2005,2012)$ met en évidence le rôle clé de l'inhibition, comme fonction exécutive, dans le choix sélectif d'une stratégie adaptée, à l'exclusion des autres. Les applications psychopédagogiques mettent l'accent sur l'inhibition qui est une forme de contrôle neurocognitif et comportemental qui permet aux enfants de résister aux habitudes, aux automatismes, aux tentations, distractions ou 
inférences, et de s'adapter aux situations complexes par la flexibilité (dynamique d'inhibition /activation de stratégies cognitives en compétition, voir Wilson et al., 2006, 2009, et " La course aux nombres ", [http://www.lacourseauxnombres.com/nr/home. php]). Le défaut d'inhibition pourrait expliquer des difficultés d'apprentissage et d'adaptation tant cognitives que sociales (Brault Foisy \& Masson, 2011).

\section{Apprentissage de la lecture}

Des études par TEP scan montrent que chez les adultes alphabétisés, la lecture de l'écriture alphabétique, comme le langage parlé, est latéralisée dans l'hémisphère gauche dans les zones occipitales, temporales et pariétales (Pugh et al., 2001). L'activité électrophysiologique de ces zones augmente avec les compétences de lecture, et est moindre chez les enfants ayant une dyslexie développementale (en anglais : developmental dyslexia ou reading disorders). Ces résultats de neuroimagerie ne conduisent pas à des applications directes mais ont permis d'éclairer les diagnostics de dyslexie basés sur le seul comportement qui restaient ambigus (Black, Myers, \& Hoeft, 2015) et ainsi d'étayer ou d'abandonner des théories controversées. Dans la dyslexie, les travaux de neuropsychologie sont en faveur d'un dysfonctionnement de systèmes neuronaux qui participent à l'acquisition normale de la lecture et d'autres fonctions cognitives associées. Lors d'exercices phonologiques les aires du langage sont insuffisamment activées chez le dyslexique. Il existe aussi la possibilité que des mécanismes cérébraux de la vision soient perturbés. La dyslexie pourrait donc être le résultat du déficit d'un ensemble de systèmes basé sur une incapacité fondamentale du cerveau à exécuter des taches demandant l'intégration de stimuli courts arrivant en succession temporelle rapide. Cette théorie de la « perturbation dans l'exécution temporelle » pourrait aussi expliquer certains symptômes perceptuels, moteurs ou cognitifs très souvent associés à la dyslexie et qui sont inexpliqués jusqu'à présent (Habib, 2000). Les expériences montrent qu'une remédiation orientée de la lecture augmente l'activité dans les aires du langage. Un entraînement phonologique intensif de quelques semaines (Joly-Pottuz \& Habib, 2008), focalisé sur le système déficient, non seulement réactive les zones affaiblies mais sollicite des zones « muettes » des deux hémisphères. Donc l'entraî- nement (une intervention extérieure) peut modifier l'organisation cérébrale dans le sens probable d'une meilleure connectivité entre des zones habituellement inutilisées.

D'autres études montrent une implication importante des aires cérébrales auditives dans la lecture ce qui a amené les chercheurs à utiliser des programmes d'entraînement auditif qui peuvent conduire à une amélioration marquée de la lecture (Temple et al., 2003).

\section{Apprentissage des mathématiques}

Concernant les mathématiques, les neurosciences cognitives montrent qu'il y aurait plus d'un système neural pour la représentation des nombres. On trouve un système de "sens du nombre » «phylogénétiquement » ancien chez les animaux ainsi que chez les enfants et adultes ; il est à la base de la connaissance des nombres et de leurs relations (Dehaene, DehaeneLambertz, \& Cohen, 1998; Ansari Fugelsang, Dhital, \& Venkatraman, 2006). Ce système, localisé de façon bilatérale dans les zones intrapariétales, est activé quand les participants effectuent des taches comme des comparaisons de nombres, nombres arabes, ensembles de points, ou nom de nombres.

Un système différent de connaissances numériques pourrait être localisé verbalement dans les aires du langage, où l'on trouve aussi un stockage de poésies et de séquences verbales apprises par cœur ${ }^{8}$. En mathématiques, il contiendrait les tâches de comptage et les tables de multiplication. Ce système linguistique serait plus impliqué dans le stockage des « faits numériques » que dans la réalisation des calculs.

Les calculs plus complexes pourraient impliquer des régions visuospatiales, ce qui attesterait de l'importance de l'imagerie mentale visuelle dans les opérations à plusieurs nombres entiers.

Enfin, une zone prémotrice pariétale est activée lorsque l'on compte sur ses doigts et lorsque l'on effectue des calculs. S'il en était ainsi, il est possible que compter sur ses doigts pendant l'enfance puisse favoriser le développement de cette zone, système de comptage qui devrait donc être encouragé à l'école.

La dyscalculie développementale est définie en neuropsychologie comme une difficulté au niveau des performances en mathématiques résultant d'un déficit situé dans les parties du cerveau qui sont 
impliquées dans le traitement du calcul. Comme pour la dyslexie, le but des chercheurs en neurosciences cognitives est de développer à terme la possibilité d'établir un diagnostic de dyscalculie basé sur le fonctionnement du cerveau, et ce dès le plus jeune âge du sujet. L'objectif est de développer également des techniques de prévention et de rééducation basées, ici encore, sur le fonctionnement du cerveau.

L'étude des troubles génétiques et développementaux associés à la dyscalculie (syndrome de Turner et syndrome d'alcoolisme fotal), montre des atteintes cérébrales dans des aires du cerveau qui sont connues pour être impliquées dans le traitement du calcul (parties spécifiques des lobes pariétaux). De plus, les enfants dyscalculiques montrent des difficultés dans des tâches cognitives de base dont on sait qu'elles activent ces aires cérébrales (Landerl et al., 2004). Comme dans le cas de la dyslexie, des remédiations sont utilisées par entraînement auditif et par des jeux (Wilson et al., 2006 ; Wilson, 2011).

Les résultats que nous avons présentés dans ce paragraphe ont été obtenus grâce à des enregistrements individuels d'un nombre de sujets suffisant pour que les données statistiques obtenues soient significatives. Ils donnent des réponses aux questions que nous avons posées quant aux représentations de la mémoire, perception et émotions dans le cerveau, et quant aux relations entre cognition et émotion. Ces données mettent en relation des potentialités de l'activité de penser et d'apprendre avec des substrats cérébraux. Elles permettent de préciser des théories sur l'apprentissage. Cependant les chercheurs en neurosciences de l'éducation vont encore plus loin en faisant l'hypothèse implicite que stimuler ou modifier les structures cérébrales adéquates va modifier, ici améliorer, les performances. La vérification de cette hypothèse sur le sujet humain pose néanmoins la question des présupposés épistémologiques qui justifient ce passage à la mise en pratique.

\section{Présupposés épistémologiques des neurosciences appliquées à l'éducation}

Les opposants aux applications à l'éducation des résultats des neurosciences cognitives portent de nombreuses critiques sur les méthodes employées qui ne prendraient pas en compte tous les paramètres relatifs à ce domaine des sciences sociales, en particulier en travaillant sur des ensembles de sujets trop restreints, en ayant des contrôles inadéquats, et en donnant des résultats pouvant être interprétés de façons différentes. Brook (2008) souligne ainsi que les sciences cognitives posent des questions centrales telles que ce qu'est un être humain, si nous possédons un libre arbitre, ainsi que sur les frontières entre notre être conscient et notre être inconscient. Il signale que toute la base conceptuelle qui sous-tend les neurosciences cognitives est fragile. Chacun des champs intervenant dans le domaine de la cognition peut avoir plusieurs interprétations, comme c'est par exemple le cas de l'attention, de l'information et de l'intelligence. Si un expérimentateur n'interroge pas ses présupposés conceptuels, ou ne considère pas la nature générale de ce qu'il fait, cela peut avoir pour conséquence que ses interprétations ne soient pas adaptées au contexte de la question.

Nous pouvons ajouter deux autres limitations aux remédiations qui pourraient être réalisées par des interventions directes sur le cerveau :

- les partisans des neurosciences éducatives font l'hypothèse implicite que les remédiations proposées ne provoqueraient pas d'effets contraires ou négatifs. Les chercheurs mentionnent seulement que, dans le cas de stimulations, elles sont non-invasives et non-douloureuses. Cependant, hormis la fonction testée (par exemple la mémoire ou l'apprentissage) et malgré quelques contrôles (recherche de troubles de l'humeur ou du comportement), il n'est pas possible de vérifier que toutes les autres fonctions du sujet restent inchangées. Ce point est d'autant plus crucial que nous avons vu dans ce chapitre que certaines zones cérébrales pouvaient traiter au moins deux fonctions (ainsi logique et émotion ; Brunetti et al., 2014) ;

- les potentialités du cerveau seraient aussi implicitement illimitées et banalement sousemployées dans les conditions normales. Ce concept s'appuie sur la théorie généralement admise de la réserve cérébrale et cognitive (Marx, 2013). Un déficit d'apprentissage chez un sujet pourrait ainsi être corrigé et augmenté par une stimulation adéquate.

Malgré ces restrictions possibles, certains auteurs (Flöel et al., 2008 ; Snowball et al., 2013) suggèrent d'étendre l'utilisation de méthodes améliorant la cognition chez des sujets ayant subi un accident vasculaire cérébral, à des individus sains. Campbell (2011) justifie la création de l'Engrammetron (labo- 
ratoire de neuroscience de l'éducation, université Simon Fraser, USA) avec les arguments suivants : - être capable de mieux comprendre si les apprenants ont appris correctement ; - augmenter les capacités d'apprentissage par des méthodes de biofeedback (ou autres); - pouvoir identifier des états mentaux favorables à l'apprentissage tels que : la mémorisation, la concentration ou l'attention sélective, la pensée critique et d'autres formes de raisonnement, et développer des pédagogies qui permettraient de les reconnaître et de les provoquer.

Nous pouvons constater que certains de ces espoirs semblent nier, ou du moins passer sous silence, le rôle des éducateurs dans le développement des qualités intellectuelles de l'enfant à travers un engagement actif et réflexif sur le contenu académique (Baehr, 2013). Cela pose donc la question de déterminer s'il faut développer chez l'enfant des qualités intellectuelles ou des standards académiques. Le présupposé épistémologique est l'identification du sujet aux performances localisables et mesurables de son cerveau, c'est à un tel sujet que s'adressent les neurosciences de l'éducation autant qu'elles participent à le construire (Caussidier, 2014). Qu'en est-il plus spécifiquement des travaux en neurodidactique des sciences?

\section{LA QUESTION DU SUJET DANS LES ÉTUDES DE NEURODIDACTIQUE DES SCIENCES}

$\mathrm{Au}$ sein des recherches en neuroéducation, dont nous venons de donner un aperçu, un champ de questionnement se singularise depuis quelques années : il s'agit de la neurodidactique. Le terme neurodidactique (neurodidactics en anglais) a été créé par le chercheur allemand en sciences de l'éducation, Gerhard Preiss (1998). Ce terme désignait la mise en place d'une approche de l'éducation basée sur les relations entre les processus d'apprentissage avec d'une part les stades de développement du cerveau, et d'autre part, les conditions environnementales pratiques de cet apprentissage. Selon Preiss, la neurodidactique a pour but d'étudier les conditions nécessaires pour que l'apprentissage soit optimisé de façon maximale. Ce terme désigne aussi maintenant le champ de recherche visant à comprendre les bases biologiques des concepts propres à la didac- tique (concepts de changement conceptuel, d'obstacle, etc.) dont elle désire identifier les localisations cérébrales dans un but de modulation. La neurodidactique propose donc des concepts généraux pour l'éducation et se décline aussi en différentes didactiques selon les disciplines (français, musique, sport, etc.). Nous nous intéresserons plus particulièrement à la didactique des sciences. La neurodidactique des sciences est définie comme « un domaine de recherche de la neurodidactique qui étudie les mécanismes cérébraux liés à l'apprentissage et à l'enseignement des sciences » (Masson, 2007). Ce domaine de recherche vise à appliquer le paradigme neuropsychologique de l'existence de corrélations entre activités neurophysiologiques et modules mentaux, aux apprentissages scolaires. Notre objectif est de caractériser, pour pouvoir les discuter ultérieurement au regard de la didactique classique, socioconstructiviste et « aneurale » des sciences, les épistémologies mises en œuvre dans le champ de la neurodidactique des sciences. Nous tenterons d'identifier:

- les enjeux éducatifs explicités ;

- les cadres théoriques mobilisés (notamment les théories relatives au sujet et les définitions de l'apprentissage) ;

- les méthodes de recherche mises en œuvre.

\section{Une neurodidactique au service de quels enjeux éducatifs?}

Dans les travaux que nous avons analysés, les enjeux des recherches en neurodidactique des sciences résident essentiellement dans la compréhension des processus cognitifs impliqués dans des apprentissages conceptuels, compréhension qui devrait permettre de rendre plus efficientes les méthodes d'enseignement. Il s'agit par exemple « d'approfondir nos connaissances sur la nature des processus cognitifs impliqués dans des tâches scolaires, d'étudier ce qui se produit dans le cerveau des élèves lors des apprentissages, de mieux comprendre les causes des difficultés particulières de certains élèves et même d'évaluer l'impact de différentes méthodes d'enseignement sur le cerveau » (Neuroéducation Québec, 2011). Ce sont donc les conditions d'acquisition de nouveaux concepts qui sont travaillées. Les questions relatives aux représentations épistémologiques ou historiques que les élèves ou les enseignants ont sur les sciences ou relevant du courant s'interrogeant sur l'apprentis- 
sage de la «nature of science» ne sont pas envisagées. Les travaux de neurodidactique des sciences ne s'intéressent pas non plus aux questions relatives aux démarches et aux méthodes de raisonnement scientifique (problématisation, investigation, expérimentation...) ni aux questions scientifiques socialement vives (ou socioscientific issues) et plus généralement aux relations entre sciences et sociétés, ni aux « éducations à » la santé, l'environnement, la citoyenneté.

Autrement dit, les enjeux éducatifs dessinés par la neurodidactique des sciences se limitent à l'acquisition de nouveaux savoirs scientifiques par les élèves. Ce travail de neurodidactique, centré sur les processus psychophysiologiques impliqués dans l'acquisition de nouveaux savoirs, doit permettre de penser des stratégies pédagogiques tant en termes d'apprentissages conceptuels que de raisonnement ou d'habiletés sociales. Il en va ainsi de la possibilité pour les élèves de développer de nouvelles « habiletés neurocognitives » telle que la flexibilité ${ }^{9}$ (Houdé, 2012).

Si les travaux de neurodidactique des sciences apparaissent homogènes du point de vue des enjeux éducatifs identifiés, ils présentent cependant une plus grande diversité en termes de cadres théoriques mobilisés et de méthodes de recherche.

\section{Cadres théoriques mobilisés en neurodidactique des sciences}

Nous avons distingué, au regard des cadres théoriques mobilisés, trois grandes catégories de recherche. La première catégorie, la moins représentée, repose sur une épistémologie que nous qualifions de neuroréductionnisme interventionniste. Dans cette approche, Cohen Kadosh et al. (2010) s'interrogent sur les mécanismes neurocognitifs impliqués dans les habiletés numériques et sur les moyens de les développer. Comme nous l'avons vu dans la partie précédente sur la mémoire, leurs études évaluent les effets de stimulations cérébrales sur les apprentissages évalués par des tests cognitifs de manipulation de symboles. Elles concluent à une augmentation significative des compétences numériques chez des sujets subissant une stimulation électrique transcrânienne (supposée augmenter les substrats énergétiques disponibles) au niveau de régions (lobes pariétaux et dorsolatéraux préfrontaux) identifiées comme étant impliquées dans la mise en ouvre de calculs complexes. Le présupposé épistémologique qui sous-tend cette étude, dépasse le cadre neuropsychologique de corrélation entre activités physiologiques cérébrales localisées et fonction cognitive pour supputer une causalité entre ces catégories. Ainsi la stimulation localisée de l'activité physiologique du sujet permettrait le développement de la fonction cognitive correspondante, presque malgré lui, au même titre que la stimulation électrique d'un muscle permet sa contraction. En ce sens le sujet n'est pas acteur conscient de ses apprentissages. On peut donc aussi considérer qu'il ne s'agit pas là de neurodidactique mais de neuroéducation.

La seconde catégorie d'études repose sur l'articulation de deux cadres théoriques : celui de la neuropsychologie et celui du changement conceptuel, qui se revendique d'une épistémologie constructiviste. La majorité des travaux que nous avons analysés relève de ce double cadre théorique. Leurs auteurs proposent ainsi de préciser pourquoi les changements conceptuels peuvent être difficiles en analysant les mécanismes cérébraux qui les sous-tendent. Ils évaluent l'activité cérébrale de sujets auxquels sont soumis des données pouvant entrer en contradiction avec leurs conceptions initiales, que ce soit relativement à l'action de molécules sur la dépression, à la chute de balles de diamètres différents (Dunbar \& Stein, 2007), à la validité de montages électriques (Masson et al., 2012, 2014) ou aux masses volumiques (Lafortune, Masson, \& Potvin, 2012). Il peut aussi s'agir de préciser quels sont les mécanismes cérébraux impliqués dans la réalisation de tâches scientifiques associées avec le "sentiment de connaître » (feeling of knowing) ou dans lesquels sont impliqués des conflits cognitifs (Potvin, Masson, $\&$ Riopel, 2009 ; Turmel, Potvin, \& Masson, 2012).

D'un point de vue méthodologique, ces travaux utilisent la technique d'imagerie par résonance magnétique fonctionnelle (IRMf) et consistent en des comparaisons statistiques d'activité cérébrale de sujets soumis à des tâches cognitives contrastées dans le contexte très particulier (caractérisé notamment par un confinement et une absence de mouvements de la tête et donc de langage verbal) de l'aimant ou «tube » de neuroimagerie.

Les images produites rendent compte de la significativité statistique de l'activation spécifique des différentes régions cérébrales en présence de stimuli contrastés proposés aux sujets : animations « scientifiques » versus « naïves » (Dunbar \& Stein, 2007 ; 
Masson et al. 2012, 2014), présentation de concepts scientifiquement « corrects » versus « erronés» (Potvin, et al., 2009 ; Turmel et al., 2012), stimuli « intuitifs» versus « contre intuitifs» et évaluation des temps de réactions pour des réponses « correctes ». Dans certains cas les tests statistiques réalisés visent à comparer également les activations cérébrales locales entre des groupes d'individus " experts » et des groupes d'individus «novices» (Dunbar \& Stein, 2007 ; Masson et al. 2012, 2014) de manière à identifier des régions cérébrales spécifiquement activées par les experts scientifiques lors d'une tâche cognitive impliquant la maîtrise d'un concept scientifique. Ces travaux argumentent de la capacité des experts scientifiques à inhiber des conceptions erronées grâce à l'activation spécifique de certaines régions cérébrales connues pour leur action inhibitrice.

Sur le plan théorique cette catégorie de travaux ne parvient pas à s'émanciper d'un neuroréductionnisme parfois caricatural. C'est du moins ce que nous tenterons de discuter par la suite en précisant dans quelle mesure l'articulation entre les cadres neuropsychologiques et ceux du changement conceptuel entretient une confusion entre les niveaux mentaux et symboliques mobilisés dans l'approche des processus de conceptualisation scientifique.

Concernant la troisième et dernière catégorie d'études en neurodidactique des sciences, les travaux se revendiquent de cadres théoriques tels que la neuropsychologie cognitive ou la cognition incarnée. Il s'agit d'aborder des problématiques d'apprentissage des sciences ou des mathématiques de manière plurielle, c'est-à-dire en interrogeant leurs modalités mentales, physiologiques et comportementales. Ainsi l'étude de Campbell et al. (2009) rapporte une investigation sur la perception des images et du raisonnement en géométrie à la fois sur le plan des représentations des sujets, de leurs réactions électrophysiologiques et de leurs attitudes comportementales. Ce sont alors les corrélations éventuelles entre ces différents niveaux qui sont testées. De même, Arda Cimen et al. (2012) explorent la connexion éventuelle entre la métacognition dans l'apprentissage des mathématiques (théorie des nombres) et des mesures physiologiques (pistage des mouvements de l'œil, électro-encéphalographie, électrooculographie, réponse cardiovasculaire), de manière à tester une éventuelle « inscription corporelle de la cognition et de l'apprentissage ». Allaire-Duquette et
Charland (2012) testent l'influence de la contextualisation des problèmes de physique mécanique sur les réactions émotionnelles des sujets.

D’un point de vue méthodologique, on retrouve comme dans la catégorie précédente la mesure de paramètres neurophysiologiques (EEG) ou psychophysiologiques (réponse cardiovasculaire, activité électrodermale) chez des sujets soumis à des stimuli ou à des tâches cognitives impliquant des concepts scientifiques.

Ces derniers travaux ne postulent pas une identité entre corrélats d'activité (neuro)physiologique et apprentissages conceptuels scientifiques. Ils testent la possibilité d'une telle identité pour la discuter en prêtant en particulier attention au langage et aux effets de contexte qui les conduisent à relativiser leur propos. Ils relèvent à notre sens d'une collaboration interdisciplinaire féconde sur le plan épistémologique en évitant les écueils neuroréductionnistes ou antiréductionnistes. Ces travaux participent au développement d'une « véritable ontologie cognitive exploratoire » (Tiercelin, 2010).

\section{DISCUSSION QUANT AUX POSSIBILITÉS D'UN DiAlOGUE AVEC LES NEUROSCIENCES DE L'ÉDUCATION}

Les partis pris épistémologiques retenus dans cette dernière catégorie de travaux en neurodidactique des sciences offrent, selon nous, la possibilité d'une mise en discussion avec nos propres cadres théoriques et méthodologiques dans le champ de la didactique des sciences. Il s'agit de réinterroger conjointement sujet apprenant, cognitif et sujet biologique dans des situations d'apprentissage contextualisées, sans présager des conclusions qui pourraient en être tirées sur le plan des processus d'apprentissage comme sur le plan praxéologique. Le fait que ces travaux se présentent comme exploratoires, sans prétention prescriptive normative en termes de stratégies didactiques apparaît ainsi comme un gage d'ouverture au dialogue interdisciplinaire.

En revanche, notre analyse des cadres épistémologiques et méthodologiques mobilisés dans les premières et deuxièmes catégories de la neuroéducation et de la neurodidactique des sciences nous conduit à conclure qu'une majorité des travaux auxquels nous nous sommes référés proposent des cadres épistémologiques qui révèlent des conceptions 
du sujet en situation d'apprentissage qui ne nous semblent pas compatibles avec les cadres que nous mobilisons en didactique des sciences, et notamment avec le socioconstructivisme.

En premier lieu, ces partis pris traduisent une théorie du sujet en situation d'apprentissage fondée sur :

1) l'établissement implicite de normes (par exemple : «appris correctement ») dont la validité et les limites ne sont pas discutées.

Cette question est d'autant plus vive que les neurosciences de l'éducation s'adressent à des sujets en devenir (Favre, 2014). L'enfant-sujet est alors considéré, par des adultes, comme handicapé, ou en situation pathologique, pour certaines de ses fonctions cognitives, handicap auquel il convient de remédier. Le handicap appelé « difficultés d'apprentissage " (learning difficulties) sera évalué en fonction de normes basées sur des appréciations statistiques. Prendra-t-on alors en compte les éventuelles autres capacités de l'enfant? Par exemple, on sait que des enfants présentant un retard mental peuvent avoir des performances intellectuelles (mémoire, calcul mental) supérieures à la moyenne (Sacks, 1985) et certains grands artistes présentaient des capacités d'apprentissage limitées, prendrait-on le risque en y remédiant de diminuer ou d'éteindre leurs capacités créatrices? Devons-nous soumettre un enfant-sujet à une telle manipulation ?

2) l'amélioration des compétences par stimulation physiologique au nom de la recherche d'une performance cognitive.

Ces perspectives s'inscrivent dans le mouvement de mobilisation des biotechnologies visant à développer une humanité " augmentée », ce qui soulève bien sûr d'importantes interrogations éthiques (Simonneau, 2014). Cette conception du sujet en situation d'apprentissage pour lequel il s'agirait de stimuler physiologiquement la performance puise en partie ses racines épistémologiques dans le concept de neuroplasticité. Ainsi le rapport de l'OCDE (2007) déjà évoqué, et se présentant tout à la fois comme synthèse des études de neuroéducation déjà publiées et programme prometteur de recherche, ménage une large place à ce dernier concept. La neuroplasticité ou plasticité cérébrale rend compte des propriétés de réorganisation structurale et fonctionnelle du système nerveux et cela à différentes échelles d'organisation (du moléculaire à l'organe). Proposée comme support aux processus de mémorisation et d'apprentissage, cette plasticité peut être observée dans le cadre du développement de l'individu normal (neuro-ontogenèse) ou en situation pathologique (par exemple à la suite d'un accident vasculaire cérébral, plasticité dite réactionnelle) au cours de sa récupération fonctionnelle. Comme nous l'avons déjà souligné, dans les études relevant de la neuroéducation ou de la neurodidactique des sciences, le concept de plasticité cérébrale est mobilisé pour rendre compte des propriétés de «flexibilité » mentale du sujet en situation d'apprentissage. Or la neuroplasticité, tout à la fois modélisation et naturalisation du processus neuronal, est susceptible de véhiculer des normes idéologiques de légitimation d'un fonctionnement social qu'il est nécessaire de questionner. C'est du moins ce à quoi nous invite Malabou (2004, p 66-67) dans les termes suivants : "La plasticité cérébrale permet-elle de penser, à titre de modèle, une multitude d'interactions dans lesquels les partenaires exercent les uns sur les autres des effets de transformation à travers des exigences de reconnaissance, de non domination et de liberté ? Ou bien faut-il considérer au contraire qu'entre déterminisme et polyvalence, la plasticité cérébrale constitue la justification biologique d'un type d'organisation économique, politique et social où seul compte le résultat de l'action en tant que tel, l'efficacité, l'adaptabilité - une flexibilité à toute épreuve $^{10}$ ? » De même dans ses « études critiques du cerveau sexué », Kraus (2013, p. 702) propose de ne pas cesser d'interroger, y compris au sein de nos propres recherches, la notion consensuelle et « très séduisante » de plasticité cérébrale et le principe associé d'« idiosyncrasie individuelle » qui rend compte de la façon dont les comportements genrés se cérébralisent, dépassant ainsi à la fois les déterminismes biologiques et sociaux. Car si les notions de neuroplasticité et d'individualité sont mobilisées au sein des neurosciences, elles le sont aussi largement à l'extérieur de celles-ci, en sciences de l'éducation par exemple, pour ce qui nous intéresse. Il s'agit donc de ne pas perdre de vue l'interrogation essentielle sur les types de sujets cérébraux produits ou non par la notion de plasticité cérébrale, mais aussi « quelle espèce d'ordre social et quelles conceptions de la capacité d'agir du sujet humain se trouvent coproduites par les savoirs sur la plasticité cérébrale » (Kraus, 2013, p.706), particulièrement lorsque sont visés des impératifs de performance et de flexibilité tels qu'ils peuvent s'exprimer dans les travaux en neuroéducation et neurodidactique des sciences. 
Pour reprendre les propos de Stanislas Morel (2014), nous nous trouvons devant une situation de " médicalisation décomplexée » qui s'appuie sur la différenciation pédagogique pour proposer des diagnostics « médico-psychologiques » (Morel, 2014).

En second lieu nous discutons plus précisément de la réduction opérée par la neurodidactique sur le processus d'apprentissage conceptuel. Il s'agit en fait à notre sens d'une double réduction. On pourrait dire qu'une première réduction est opérée par la visée didactique elle-même, au nom d'une finalité sociale qui est de faire évoluer les conceptions des apprenants. Nous ne revenons pas sur ce débat qui a conduit entre autres raisons à distinguer les notions de représentation et de conception ${ }^{11}$. La seconde réduction, propre à la neurodidactique, est une forme de réductionnisme matérialiste que nous allons expliciter.

Ce réductionnisme, la réduction des processus d'apprentissage à leurs corrélats d'activité physiologique, repose fondamentalement sur une entrée dans la compréhension des processus d'apprentissage conceptuels entendus comme fonctions, avec un lien causal entre structures cérébrales et fonctions. On retrouve là une confusion entre carte et territoire. Cette confusion se double souvent d'une confusion entre ce qu'est le concept en jeu dans l'apprentissage (ce qui relève d'un problème ontologique) et la façon dont le cerveau se représente ce concept (ce qui relève d'un problème épistémologique). Plus précisément, il nous semble que les travaux de neurodidactique des sciences qui intègrent le cadre du changement conceptuel proposent d'ouvrir la boîte noire des processus cognitifs mentaux (ou structure cognitive) mais à l'aide de paradigmes expérimentaux mobilisant des niveaux symboliques. C'est dans ce sens qu'ils opèrent une confusion entre le niveau mental des concepts spontanés individuels inconscients et le niveau symbolique des concepts scientifiques, construits socialement, explicites et communicables. Cette distinction essentielle a été introduite par Vygotsky (1986 [1934]). Dans sa clarification sur les usages du (socio)constructivisme en didactique des sciences. Bächtold (2013) montre qu'elle n'est pas toujours explicitée. Au sein $\mathrm{du}$ réductionnisme neurodidactique, cette confusion entre niveaux mentaux et symboliques apparaît permanente et constitutive.

S'il nous semble important de clarifier les fondements d'un tel neuroréductionnisme c'est parce qu'ils nous permettent de comprendre la tendance des études de neurodidactique à essentialiser la question des apprentissages scientifiques, notamment en faisant l'impasse à la fois sur la diversité des enjeux éducatifs auxquels ils participent et sur les contextes socioculturels dans lesquels ils s'inscrivent. Certes, certains neuroéducateurs (Lalancette \& Campbell, 2012) s'interrogent sur les contextes extrêmement contraints des études mobilisant les techniques d'IRMf notamment, où le sujet est seul, confiné dans un tube sans possibilités de mouvements de la tête et donc sans interactions langagières verbales possibles avec d'autres sujets. Mais c'est pour mieux inviter à les dépasser dans une réflexion neuroéthique plus générale ${ }^{12}$.

Or nous sommes bien loin, dans ces conditions expérimentales, des contextes authentiques d'apprentissage. À rebours de la didactique des sciences qui a progressivement intégré les contextes sociaux et culturels (pratiques sociales de références, conceptions situées : savoirs, valeurs, pratiques, questions socialement vives) dans sa réflexion sur les apprentissages conceptuels de sciences, la neurodidactique opère une régression radicale. Autant dire qu'avec cette conception du sujet en situation d'apprentissage qui porte si peu attention au contexte, les perspectives de dialogue interdisciplinaire apparaissent drastiquement réduites. Que dire par exemple des contrats didactiques ou des rapports aux savoirs en jeu chez un élève placé dans un appareillage d'imagerie par résonance magnétique et à qui on propose des tests cognitifs?

L'essentialisation des processus d'apprentissage que révèle la grande majorité des approches neurodidactiques doit, en retour, nous inviter à ne pas perdre de vue l'importance de contextualiser nos propres travaux didactiques et à clarifier les enjeux éducatifs dans lesquels ils s'inscrivent. Car il nous semble que penser l'apprentissage c'est tenter de réfléchir de manière indissociable les enjeux et les contextes éducatifs, leur prise en charge par des sujets volontaires, conscients, réflexifs. Rappelant le postulat anthropologique de l'analyse de l'expérience vécue comme " communication entre l'espace du corps avec le temps de la culture », Foucault $(1966$, p. 363) envisageait dans les termes suivants ce que l'homme représente pour les sciences humaines : «Pourquoi l'anatomie des centres corticaux du langage ne peuvent en aucune manière être considérées comme des sciences de l'homme. C'est que l'objet de celles-ci 
ne se donne jamais sur le mode d'être d'un fonctionnement biologique (ni même de sa forme singulière et de son prolongement en l'homme); il en est plutôt l'envers, la marque en creux; il commence là où s'arrête non pas l'action ou les effets, mais l'être de ce fonctionnement - là où se libèrent des représentations vraies ou fausses, claires ou obscures, parfaitement conscientes ou engagées dans la profondeur de quelque somnolence, observables directement ou indirectement, offertes en ce que l'homme énonce lui-même, ou repérables seulement de l'extérieur ; la recherche des liaisons intracorticales entre les différents centres d'intégration du langage (auditifs, visuels, moteurs) ne relève pas des sciences humaines; mais celles-ci trouveront leur espace de jeu dès qu'on interrogera cet espace de mots, cette présence ou cet oubli de leur sens, cet écart entre ce qu'on veut dire et l'articulation où cette visée s'investit, dont le sujet n'a peut-être pas conscience, mais qui n'aurait aucun mode d'être assignable si ce même sujet n'avait des représentations ».

Ce à quoi Foucault nous invite, c'est à ne pas sombrer dans l'illusion d'une conception objectiviste qui fait l'impasse du sujet acteur « obligatoire » de ses propres expériences, qui l'en dépossède en quelque sorte, dans l'accès que l'on peut avoir scientifiquement à ces expériences. Les partis pris épistémologiques des neurosciences de l'éducation et de la neurodidactique nous conduisent ainsi à réaffirmer quelles sont les théories du sujet que nous mobilisons dans nos approches scientifiques des expériences d'apprentissage. D'autres domaines de recherche ont été confrontés à cette exigence. Ainsi, par exemple, la recherche clinique sur les troubles mentaux où des chercheurs tels que Bovet et Parnas (2013) opposent au développement des approches objectivistes portées par les neurosciences, une clinique phénoménologique du sujet.

Pour notre part nous faisons le constat que la neurodidactique des sciences, qui s'inspire beaucoup de la neuroéducation, apparaît polarisée par deux orientations. La première considère l'apprentissage sous l'angle des difficultés qui lui sont afférentes, envisagées comme des pathologies. Les enseignants et chercheurs auraient alors le devoir d'intervenir pour remettre le sujet apprenant dans la «normalité ». La deuxième est celle qui leur demanderait de répondre à une demande sociale. Ainsi, aux ÉtatsUnis, les partisans d'une éducation sexuelle basée sur l'abstinence font référence aux études de neuros- ciences pour conclure que " the healthiest behavior, both physically and emotionally, is for persons to abstain from sex until they can commit to one partner for the rest of their lives " (Ballonoff Suleiman et al., 2015). L'orientation socioconstructiviste qui est la nôtre nous conduit à argumenter sur la dimension normative de ces recherches neurodidactiques et à souligner les limites, voire les dangers des pratiques d'enseignement qu'elles peuvent légitimer.

Mais le dialogue interdisciplinaire est ouvert et il est possible d'inviter les neuroscientifiques qui prennent les apprentissages et l'éducation comme objets à envisager une épistémologie phénoménologique. Comme il apparaît d'ailleurs fructueux d'interroger en retour les chercheurs en sciences de l'éducation et en didactique sur le sujet (neuro)biologique qui participe de cette phénoménologie. C'est du moins ce que nous avons tenté de faire.

Finalement, au regard des réductionnismes opérés au sein des champs de la neuroéducation et de la neurodidactique des sciences, comme au regard des dérives normatives probables annoncées par la mobilisation des notions de réhabilitation, d'amélioration, de flexibilité et de performance dans le domaine des apprentissages, nous ne pouvons que souligner avec Harrison (2008) l'importance du « travail conceptuel à mener avant de faire chauffer les scanners ». Pour autant, cette forme de vigilance épistémologique ne doit pas altérer la volonté de tisser un dialogue interdisciplinaire fructueux pour une prise en compte renouvelée des dimensions cognitives, sociales et biologiques du sujet en situation d'apprentissage. Cette perspective invite par exemple à entrer dans le débat pour discuter les dualismes nature / culture, biologique / social souvent mobilisés. Ce débat doit nous permettre de préciser nos conceptions du sujet en situations d'apprentissage, les catégories d'analyse que nous utilisons (mental versus symbolique par exemple), mais aussi l'attention que nous prêtons ou non aux questions de contextes et plus fondamentalement aux enjeux d'enseignement. 


\section{NOTES}

1. C'est d'ailleurs autour de cette diversité de problématiques que la Maison des sciences de l'homme Paris Nord a organisé en 2012, une journée d'étude intitulée « Les usages sociaux des sciences du cerveau ». De même en 2013, la Revue d'Anthropologie des Connaissances proposait un numéro intitulé « Les neurosciences à l'épreuve de la clinique et des sciences sociales » consacré aux neurosciences sociales ou social neurosciences.

2. En 2013, le service de veille et d'analyse de l'Institut français d'éducation produisait un rapport intitulé "Neurosciences et éducation : la bataille des cerveaux". Les objectifs des auteurs de ce rapport étaient les suivants : « interrogeant le point de vue qui considère les neurosciences de l'éducation comme une science qui va radicalement bouleverser nos manières d'apprendre, voire d'enseigner, nous avons souhaité dans ce dossier mettre en perspective ce que l'on sait aujourd'hui entre cerveau et école ». Il s'agit d'évaluer si les neurosciences de l'éducation sont « capables de rendre les pratiques enseignantes plus efficaces et d'aider les élèves à mieux apprendre » (Gaussel \& Reverdy, 2013, p. 1).

3. Nous focalisons la réflexion sur la didactique des sciences car il s'agit du domaine de recherche actuel des deux auteurs. Par ailleurs : Claude Caussidier est docteur d'État en neurosciences et a réalisé la majorité de ses travaux de recherche dans ce domaine au sein de l'unité 432 de l'Inserm ; Grégoire Molinatti a une formation initiale en neurosciences puis il a soutenu une thèse en sciences de la communication et en didactique des sciences, sur la thématique de la médiation des implications sociales du développement des neurosciences (Museum national d'histoire naturelle). Par ailleurs, il a coordonné le développement d'un logiciel de visualisation et d'une banque de données de neuroimagerie, à visée éducative (voir Monod-Ansaldi et al., 2012).

4. Traduction des auteurs : " the cerebellum is the primary seat of the rational soul in man, and of the sensitive soul in animals".

5. Le titre est emprunté au rapport de l'OCDE publié sur le sujet en 2007.

6. O. Houdé, instituteur de formation initiale, est professeur de psychologie à l'université Paris Descartes (Sorbonne) et directeur du LaPsyDÉ (CNRS). Il est spécialiste du développement de l'intelligence chez l'enfant et des apprentissages (psychopédagogie expérimentale).

7. Pour un aperçu des fondements méthodologiques de l'IRMf : [http://acces.ens-lyon.fr/acces/thematiques/ neurosciences/actualisation-des-connaissances/methodes_ etude_cerveau/irm_atomique_et_fonctionnelle/ irm_generalites/generalites-sur-lirm].

8. D'autres modèles (pour une revue voir Cerles, 2015) «...proposent l'existence de traces mnésiques multimodales comprenant de nombreux composants de type visuels, émotionnels, spatiaux, linguistiques, etc. La récupération épisodique consiste alors à simuler ces différents composants ensemble ou plus largement à simuler les opérations effectuées à l'encodage (par activation de traces). Par exemple, la remémoration d'un stimulus visuel consistera en la réactivation des aires visuelles activées lors de sa présentation à l'apprentissage. De même, la présentation du mot mouton activera par simulation les aires sensorimotrices activées lors d'expériences précédentes avec cet animal, pouvant entrainer le rappel d'une expérience particulière »(Cerles, 2015, p. 295).

9. « Cette nouvelle conception du développement cognitif ouvre la voie à des applications psychopédagogiques. L'inhibition est, en effet, une forme de contrôle neurocognitif qui permet aux enfants de résister aux habitudes ou automatismes, aux tentations, distractions ou interférences, et de s'adapter aux situations complexes par la flexibilité. Le défaut d'inhibition peut expliquer des difficultés d'apprentissage (erreurs, biais de raisonnement, etc.) et d'adaptation tant cognitive que sociale » (Houdé, 2012). 10. Dans la perspective du développement d'un libre arbitre, Malabou (2004) appelle à revendiquer de savoir, à travers la mobilisation du concept de plasticité, « ce que le cerveau peut faire et pas seulement tolérer. Par le verbe "faire", nous n'entendons pas seulement "faire" des mathématiques ou du piano, mais bien faire son histoire, devenir le sujet de son histoire, saisir le lien entre la part de nondéterminisme génétique à l'œuvre dans la constitution du cerveau et la possibilité d'un non-déterminisme social et politique, en un mot d'une nouvelle liberté, d'une nouvelle signification de l'histoire » (p. 31-32).

11. Voir à ce sujet Giordan, Girault \& Clément (1993). Le Marec (1996, p. 110) précise dans les termes suivants la spécificité des représentations sociales : "Il se trouve que la dynamique même des représentations sociales rend très difficile toute prédétermination de ce que l'on peut en tirer à partir d'une finalité sociale qui se situerait au-dessus de toutes les autres, puisque les représentations sociales sont des systèmes incluant eux-mêmes des orientations et des finalités sociales. »

12. Nous avons déjà souligné en début d'article cette caractéristique des neurosciences sociales à nourrir leur autocritique, organisée dans le courant des neurocritical science. Ce qui se présente comme une volonté de développer la conscience des chercheurs en neurosciences vis-à-vis des 
dynamiques et des contextes sociaux dans lesquels ils travaillent ou encore des implications éthiques de leurs recherches, n'apparaît souvent que comme une illusion de réflexivité. Comment interpréter autrement les recommandations présentées dans la section « Éthique et organisation de la neuroscience de l'éducation » du rapport de l'OCDE mentionné plus haut (2007, p. 143) quant aux « Risques d'une approche trop scientifique de l'éducation » (sic!).

\section{RÉFÉRENCES}

Allaire-Duquette, G., \& Charland, P. (2012). Linfluence de la contextualisation des problèmes de physique mécanique sur la réaction émotionnelle d'étudiantes $d u$ collégial : un projet de recherche exploratoire utilisant une mesure psychophysiologique. Communication au colloque organisé dans le cadre du $17^{\mathrm{e}}$ Congrès de l'AMSE du 3 au 8 juin 2012. Université de Reims, France.

Ansari, D., Fugelsang, J. A., Dhital, B., \& Venkatraman, V. (2006). Dissociating response conflict from numerical magnitude processing in the brain: An event-related fMRI study. NeuroImage, 32, 799-805.

Arda Cimen, O., Campbell, S. R., \& Pagé, P. (2012). Une étude de cas en neuroscience éducationnelle : l'apprentissage de concepts de base de la théorie élémentaire des nombres. Communication au colloque organisé dans le cadre du $17^{e}$ Congrès de l'AMSE du 3 au 8 juin 2012. Université de Reims, France.

Atherton, M (2005). Applying the Neurosciences to Educational research: Can Cognitive Neuroscience Bridge the Gap? Part 1. Paper presented at the annual meeting of the American Educational Research Association. Canada : Montreal

Baehr, J. (2013). Educating for intellectual virtues: from theory to practice. Journal of Philosophy of Education. 47(2), 248-263.

Bächtold, M. (2013). What Do Students "Construct" According to Constructivism in Science Education? Research in Science Education. 43(6), 2477-2496.

Ballonoff Suleiman, A., Johnson, M., Shirtcliff, E. A., \& Galván, A. (2015). School-Based Sex Education and Neuroscience: What We Know About Sex, Romance, Marriage, and Adolescent Brain Development. Journal of School Health, 85, 567-574. DOI : 10.1111/josh.12285.

Black, J. M., Myers, C. A., \& Hoeft, F. (2015). The utility of neuroimaging studies for informing educational practice and policy in reading disorders. New Directions for Child and Adolescent Development, 147, 49-56.
Bovet, E., Kraus, C., Panese, F., Pidoux, V., \& Stücklin, N. (2013). Les neurosciences à l'épreuve de la clinique et des sciences sociales. Revue d'anthropologie des connaissances, 7(3), 555-569.Bovet, P., \& Parnas, J. (2013). Quelle épistémologie pour les neurosciences en psychiatrie ? Revue d'anthropologie des connaissances. 7(3), 655-666.

Boyd, L. A., \& Linsdell, M. A. (2009). Excitatory repetitive transcranial magnetic stimulation to left dorsal premotor cortex enhances motor consolidation of new skills. BMC Neuroscience, 10(72), 75-84.

Brault Foisy, L.-M., \& Masson, S. (2011). Apprendre les sciences, c'est apprendre à inhiber ses conceptions antérieures ? Spectre, 40(2), 30-33.

Brook, A. (2008). Philosophy in and Philosophy of Cognitive Science. Dans K. Appiah (Ed.), Experiments in Ethics. Cambridge, MA : Harvard University Press.

Brousseau, G. (1998) Théorie des situations didactiques. Grenoble : La Pensée Sauvage.

Brunetti, M., Perrucci, M. G., Di Naccio, M. R., Ferretti, A., Del Gratta, C., Casadio, C., \& Romani, G. L. (2014). Framing deductive reasoning with emotional content: An fRMI study. Brain and Cognition. 87, 153-160.

Campbell, S. R. (2011). Educational neuroscience: motivations, methodology, and implications. Educational Philosophy and Theory. 43(1), 7-16.

Campbell, S. R., Handscomb, K., Zaparyniuk, N. E., Sha, L., Cimen, O. A., \& Shipulina, O. V. (2009). Investigating image-based perception and reasoning in geometry. Paper presented to the American Educational Research Association : Brain, Neuroscience, and Education SIG (San Diego, CA).

Canguilhem, G. (1989 [1949]). La connaissance de la vie. Paris : Vrin.

Caussidier, C. (2014). Le sujet neurocognitif et l'éducation : un paradoxe ? Éducation et Socialisation $n^{0} 16$, Dossier 2 : Le sujet dans les éducations à... Repéré à : [http://edso.revues.org/872].

Cerles, M. (2015). Évaluation et développement d'un modèle de la mémoire épisodique reposant sur un processus de mise à jour égocentrée. Médecine humaine et pathologie. Université Grenoble Alpes. Français : <NNT : 2015GREAS003>. Repéré à : [https://tel. archives-ouvertes.fr/tel-01179835/document].

Changeux J.-P. (1983). L'homme neuronal. Paris : Fayard. Chevallard, Y. (1985). La transposition didactique. Grenoble: La pensée sauvage.

Clément, P. (2004). Sciences et idéologie : exemples en didactique et épistémologie de la biologie, Actes du Colloque Sciences, médias et société. Lyon, 15-17 juin 2004. École normale supérieure lettres et sciences humaines, p. 53-69. 
Cohen Kadosh, R., Soskic, S., Iuculano, T., Kanai, R., $\&$ Walsh, V. (2010). Modulating Neuronal Activity Produces Specific and Long-Lasting Changes in Numerical Competence. Current Biology. 20(22), 2016-2020.

Damasio, A. R. (2001). L'erreur de Descartes. Paris : Odile Jacob.

Dehaene, S. La course au nombre. Repéré à : [http:// www.lacourseauxnombres.com/nr/home.php] (consulté le 14/04/2016).

Dehaene, S., Dehaene-Lambertz, G., \& Cohen, L. (1998). Abstract representations of numbers in the animal and human brain. Trends in Neuroscience, 21(8), 355-611.

Dunbar, K., Fugelsang, J., \& Stein, C. (2007). Do naive theories ever go away? Using brain and behavior to understand changes in concepts. Dans M. Lovett $\&$ P. Shah (Eds.), Thinking with data (p. 193-206). New York : Lawrence Erlbaum Associates.

Edelman, G. (1987). Neural Darwinism: The Theory of Neuronal Group Selection. New York : Basic Books.

Eustache, F., \& Guillery-Girard, B. (2016). La neuroéducation. La mémoire au cour des apprentissages. Paris : Odile Jacob.

Favre, D. (2014). Sur les traces d'un « sujet en devenir ». Éducation et Socialisation $n^{\circ} 16$, Dossier 2 : Le sujet dans les éducations à... Repéré à : [http://edso. revues.org/1058].

Flöel, A., Rösser, N., Michka, O., Knecht, S., \& Breitenstein, C. (2008). Noninvasive Brain Stimulation Improves Language Learning. Journal of Cognitive Neuroscience, 20(8), 1415-1422.

Foucault, M. (1966). Les mots et les choses : une archéologie des sciences humaines. Paris: Gallimard.

Gaussel, M., \& Reverdy, C. (2013). Neurosciences et éducation: la bataille des cerveaux. Dossier d'actualité $\mathrm{n}^{\circ}$ 86. Lyon : École normale supérieure de Lyon.

Giordan, A., Girault, Y., \& Clément, P. (Ed.) (1993). Conceptions et connaissances, Berne : Peter Lang.

Girotto, V., \& Gonzalez, M. (2008). Children's understanding of posterior probability. Cognition, 106, 325-344.

Habib, M. (2000). The neurological basis of developmental dyslexia. An overview and working hypothesis. Brain, 123(12), 2373-2399.

Hardiman, M., Rinne, L., Gregory, E., \& Yarmolinskaya, J. (2011). Neuroeducation and Classroom Teaching: Where the Brain Sciences Meet Pedagogy Neuroethics. DOI : 10.1007/s12152-011-9116-6.

Houdé, O. (2005). Se développer s'est apprendre à inhiber. La Recherche, 388, 74-78.Houdé, O., Zago, L., Crivello, F., Moutier, S., Pineau, A., Mazoyer, B., \& Tzourio-Mazoyer, N. (2001). Access to deductive logic depends on a right ventromedial prefrontal area devoted to emotion and feeling: evidence from a training paradigm. NeuroImage, 14, 1486-1492.

Houdé, O., Pineau, A., Leroux, G., Poirel, N., Perchey, G., Lanoë, C., Lubin, A., Turbelin, M.-R., Rossi, S., Simon, G., Delcroix, N., Lamberton, F., Vigneau, M., Wisniewski, G., Vicet, J.-R., \& Mazoyer, B. (2011). Functional magnetic resonance imaging study of Piaget's conservation-of-number task in preschool and school-age children: A neo-Piagetian approach. Journal of Experimental Child Psychology, 110, 332-346.

Houdé, O. (2012). Inhibition et correction d'erreurs dans le cerveau humain. Communication présentée au Troisième colloque de Neuroéducation Québec (en collaboration avec l'EREST) réalisé dans le cadre du $17^{\mathrm{e}}$ Congrès international de l'Association mondiale des sciences de l'éducation, université de Reims, France.

Iuculano, T., \& Cohen Kadosh, R. (2013). The Mental Cost of Cognitive Enhancement. The Journal of Neuroscience, 33, 4482-4486.

Jacob, F. (1981). Le jeu des possibles, Essai sur la diversité du vivant. Paris : Fayard.

Jacobson, L., Goren, N., Lavidor, M., \& Levy, D. A. (2012). Oppositional transcranial direct current stimulation (tDCS) of parietal substrates of attention during encoding modulates episodic memory. Brain Research, 23, 66-72.

Joly-Pottuz, B., \& Michel Habib, M. (2008) Au-delà des entraînements phonologiques de la dyslexie : traitement visuo-attentionnel versus stimulation intermodalitaire. Revue de Neuropsychologie, 18, 327-365.

Joshua, S., \& Dupin, J.-J. (1989). Représentation et modélisation : le débat scientifique dans la classe et l'apprentissage de la physique. Berne : Peter Lang.

Kraus, C. (2013). Études critiques du cerveau sexué. Une critique de quoi et pour qui ? Revue d'anthropologie des connaissances, 7(3), 693-716.

Lafortune, S., Masson, S., \& Potvin, P. (2012). Étude du développement cérébral de la capacité à surmonter les interférences intuitives en sciences. Communication au colloque organisé dans le cadre du $17^{\mathrm{e}}$ Congrès de l'AMSE du 3 au 8 juin 2012. Université de Reims, France.

Lalancette, H., \& Campbell, S. R. (2012). Educational neuroscience: Neuroethical considerations. Int. Journal of Environmental \& Science Education, 7(1), 37-52.

Le Marec, J. (1996). Le visiteur en représentations. L'enjeu des évaluations préalables en muséologie (Thèse de sciences de l'information et de la communication). Université Jean Monnet de Saint-Étienne.

Lemerle, S. (2011). «Une nouvelle "lisibilité du monde" : les usages des neurosciences par les intermédiaires culturels en France (1970-2000) », Revue 
d'Histoire des Sciences Humaines, 2(25), 35-58. DOI : 10.3917/rhsh.025.0035.

Malabou, C. (2004). Que faire de notre cerveau? Paris: Bayard.

Marx, P. (2013). Mentally fit. Workouts at the brain gym. The New Yorker, 29 juillet, 24-28.

Masson, S., Potvin, P., Riopel, M., \& Brault-Foisy, L. M. (2014). Differences in Brain Activation Between Novices and Experts in Science During a Task Involving a Common Misconception in Electricity. Mind, Brain, and Education, 8(1), 44-55.

Masson, S., Potvin, P., Riopel, M., Brault-Foisy, L.-M., \& Lafortune, S. (2012).Using fMRI to study conceptual change: Why and how? International Journal of Environmental and Science Education, 7, 19-35.

Masson, S. (2007). Enseigner les sciences en s'appuyant sur la neurodidactique des sciences. Dans P. Potvin, M. Riopel \& S. Masson (dir.), Enseigner les sciences: regards multiples (p. 308-321). Québec : Éditions MultiMondes. Accessible en ligne (site consulté le 22 mai 2014) : [http://labneuroeducation.org/s/ Masson2007.pdf].

Molinatti, G. (2007). Médiation des sciences du cerveau : approche didactique et communicationnelle de rencontres entre neuroscientifiques et lycéens (Thèse de doctorat en muséologie, sciences sociétés, Muséum national d'histoire naturelle). Paris.

Monod-Ansaldi R., Molinatti, G., Fontanieu V., Devallois, D., \& Sanchez, E. (2012). Tests statistiques et IRM cérébrales en classe de première $S$. RDST, 5, 159-184.

Morel, S. (2015). Regard sociologique sur la médicalisation de l'échec scolaire. Lecture-Éducation-2015. Entretien IRESMO. Propos recueillis par Irène Pereira. Disponible en ligne (site consulté le 26 mars 2015) : [http://iresmo.jimdo.com/2015/01/04/regardsociologique-sur-la-m\%C3\%A9dicalisation-de-l\%C3\%A9chec-scolaire/].

Neuroéducation Québec (2011). Description du deuxième colloque scientifique organisé par Neuroéducation Québec présenté dans le cadre du $79^{e}$ Congrès de l'Acfas. 12 mai 2011. Disponible en ligne (site consulté le 22 mai 2014) : [http://www.associationneuroeducation.org/colloques? category=Colloque $\% 20$ scientifique\%202011].

OCDE, Organisation de coopération et de développement économique (2007). Comprendre le cerveau : naissance d'une science de l'apprentissage. OECD Publishing. Disponible en ligne (site consulté le 22 mai 2014) : [http://www.oecd.org/ $\mathrm{fr} /$ sites/educeri/comprendrelecerveaunaissancedunesciencedelapprentissage.htm].
Piaget, J. (1192, [1967]). Biologie et connaissance : essai sur les relations entre les régulations organiques et les processus cognitifs. Delachaux et Niestlé: Neuchâtel.

Posner, G. J., Strike, K. A., Hewson, P. W., \& Gertzog, W. A. (1982). Accommodation of a scientific conception: Toward a theory of conceptual change. Science Education, 66, 211-227.

Posner, M. I., Rothbart, M. K., \& Rueda, R. (2011). Brain mechanisms and learning of high level skills. En ligne (Consulté le 26 septembre 2012) : [http://www.bowdoin.edu/ sputnam/rothbarttemperament-questionnaires/cv/publications/ pdf/2005-brain-mech-learning-high-level-skillsposner-rothbart-rueda.pdf].

Potvin, P., Masson, S., \& Riopel, M. (2009). Étude neuroscientifique $d u$ rôle du doute dans les apprentissages scientifiques qui nécessitent des changements conceptuels. Communication au premier colloque scientifique organisé par Neuroéducation Québec présenté dans le cadre du $77^{\mathrm{e}}$ congrès de l'ACFAS. 12 mai 2009, université d'Ottawa, Canada.

Preiss, G. (Ed.) (1998). Neurodidaktik. Theoretische und praktische Beiträge. Herbolzheim : Centaurus.

Pugh, K. R., Mencl, W. E., Jenner, A. R., Katz, L., Frost, S. J., Lee, J. R., Shaywitz, S. E., \& Shaywitz, B. A. (2001). Neurobiological studies of reading and reading disability. Journal of Communication Disorders, 34, 479-492.

Sacks, O. (1985/1992). L'homme qui prenait sa femme pour un chapeau. Paris : Seuil.

Sensevy, G., \& Mercier, A. (2007). Agir Ensemble L'action didactique conjointe du professeur et des élèves. France: Presses universitaires de Rennes.

Simonneau, L. (2014). Éléments pour une théorie biologique du sujet. Éducation et socialisation $n^{o} 16$, Dossier 2 : Le sujet dans les éducations à... Repéré à : [http://edso.revues.org/1032].

Simonneaux, J. (2011). Les configurations didactiques des questions socialement vives économiques et sociales (HDR). Université de Provence, Aix-Marseille, $199 \mathrm{p}$.

Snowball, A., Tachtsidis, I., Popescu, T., Thompson, J., Delazer, M., Zamarian, L., Zhu, T., \& Cohen Kadosh, R. (2013). Long-Term Enhancement of Brain Function and Cognition Using Cognitive Training and Brain Stimulation. Current Biology, 23, 987-992.

Temple, E., Deutsch, G. K., Poldrack, R. A., Miller, S. L., Tallal, P., Merzenich, M. M., \& Gabrieli, J. D. E. (2003). Neural deficits in children with dyslexia ameliorated by behavioral remediation: Evidence from functional MRI. Proceedings of the National Academy of Sciences, 100(5), 2860-2865. 
Tiberghien, G., (2007). Entre neurosciences et neurophilosophie : la psychologie cognitive et les sciences cognitives. Psychologie Française, 52, 279-297.

Tiercelin, C. (2010). Sommes-nous enfin dotés d'un cérébrascope et si oui, pour quoi faire? Collège de France, Open Edition, Paris. Disponible en ligne (site consulté le 22 mai 2014) : [http://books.openedition. org/cdf/1534?lang=en].

Turmel, E., Potvin, P., \& Masson, S. (2012). Conception et mise au point d'une tâche cognitive en électricité en vue d'une utilisation pour une recherche en IRMf. Communication au colloque organisé dans le cadre du $17^{\mathrm{e}}$ Congrès de l'AMSE du 3 au 8 juin 2012. Université de Reims, France.

Vergnaud, G. (coord.) (1994). Apprentissages et didactiques, ou en est-on? Paris: Hachette Éducation.

Vygotsky, L. (1985/1934). Pensée et langage (trad. de F. Sève). Paris: Éditions Sociales.
Wang, J. X., Rogers, L. M., Gross, E. Z., Ryals, A. J., Dokucu, M. E., Brandstatt, K. L., Hermiller, M. S., \& Voss, J. L. (2014). Science, 345(6200), 1054-1057.

Wilson, A. J., Dehaene, S., Pinel, P., Revkin, S. K., Cohen, L., \& Cohen, D. (2006). Principles underlying the design of "The Number Race", an adaptive computer game for remediation of dyscalculia. Behavioral and Brain Functions, 2, 19.

Wilson, A. J., Dehaene, S., Dubois, O., \& Fayol, M. (2009). Effects of an Adaptive Game Intervention on Accessing Number Sense in Low-SocioeconomicStatus Kindergarten Children. Mind, Brain and Education, 3, 224-234.

Wyhe, J. V. (2002). The authority of human nature: the Schädellehre of Franz Joseph Gall. British Journal for the History of Science, March, 17-42. 